\title{
Bloqueio da Hipófise com Goserelina de Depósito em Ciclos de Hiperestimulação Ovariana Controlada para Fertilização in vitro
}

\author{
Pituitary Down-Regulation with the Use of Goserelin Depot in Cycles of Controlled Ovarian \\ Hyperstimulation for in vitro Fertilization
}

Luiz Eduardo Trevisan de Albuquerque, Yaron Hameiry

Luiz Eduardo Vieira Diniz

\begin{abstract}
RESUMO
Objetivo: estudar os efeitos da goserelina de depósito como agonista do GnRH para a supressão hipofisária, durante a hiperestimulação ovariana controlada (HOC) em fertilização in vitro e transferência de embriões (FIVeTE).

Método: foram estudados prospectivamente 110 ciclos envolvendo 101 mulheres. A goserelina de depósito foi administrada em dose única subcutânea, para algumas mulheres (87 ciclos) na primeira fase do ciclo menstrual e para outras (23 ciclos), na fase lútea. A administração das gonadotrofinas de mulheres menopausadas (hMG) era diária até que fossem identificados ao menos dois foliculos com diâmetro maior ou igual a $18 \mathrm{~mm}$, quando se administrava a gonadotrofina coriônica (hCG) e programava-se a aspiração folicular. Resultados: a idade média das mulheres foi de 36,7 anos (variando de 23 a 42 anos). As principais indicações para a realização da FIVeTE foram: fator tuboperitoneal (75,2\%), endometriose $(10,9 \%)$, fator ovulatório $(7,9 \%)$, fator masculino $(3,0 \%)$ e esterilidade sem causa aparente (3,0\%). Do total de casos que iniciaram a HOC, 28 (25,5\%) foram suspensos. Em 7 ciclos (8,04\% dos casos medicados na primeira fase do ciclo menstrual), houve necessidade de se realizar a aspiração dos cistos ovarianos. Foram transferidos em média 3,3 embriões por paciente (variação de 1 a 5 embriões por paciente). Das 70 transferências de embriões realizadas, resultaram 16 gravidezes clinicas (taxa de gravidez: 22,85\%).

Conclusão: a administração da goserelina de depósito é uma alternativa útil para a supressão hipofisária em FIVeTE pois não compromete os resultados quando comparados aos observados na literatura, e não é necessário que a paciente compareça diariamente para receber a medicação, fato de extrema importância num serviço público.
\end{abstract}

PALAVRAS-CHAVE: Indução da ovulação. Gonadotrofinas. Fertilização in vitro. Infertilidade.

\section{Introdução}

Os análogos de GnRH (GnRH-a) são um dos itens mais recentes do arsenal terapêutico utilizado nas técnicas de reprodução assistida. Sua administração continuada leva a um estado

Centro de Referência da Saúde da Mulher, Nutrição, Alimentação e Desenvolvimento Infantil (CRSMNADI), Secretaria da Saúde, São Paulo, SP Correspondência:

Luiz Eduardo Trevisan de Albuquerque

Avenida Angélica, 1757 - Conjunto 73 - Higienópolis

04011-002 - São Paulo - SP induzido de dessensibilização hipofisária que se manifesta como hipopituitarismo e, conseqüentemente, hipogonadismo ${ }^{1}$.

Inicialmente, Howles et $a .^{2}$ demonstraram que a presença de niveis tônicos elevados do hormônio luteinizante $(\mathrm{LH})$ reduz os índices de sucesso de um ciclo de fertilização in vitro e transferência de embriões (FIVeTE). Procurou-se, com o uso do análogo para inibir a secreção hipofisária, melhorar o prognóstico deste grupo de mulheres. Em seguida, observou-se que muitos ciclos eram frustrados por terminarem em ovulações espontâneas, provavelmente devido aos 
níveis elevados de estradiol produzidos pelo uso do citrato de clomifeno e gonadotrofinas ${ }^{3,4}$, fazendo com que ocorresse a liberação de um pico espontâneo de LH. Por último, Pellicer et al. ${ }^{5}$ descreveram a existência de um elevado número de mulheres que não respondiam bem aos medicamentos estimulantes da ovulação (más respondedoras), mesmo com a aplicação dos protocolos de estimulação clássicos já existentes.

Nos primeiros ensaios clínicos demonstrouse que o GnRH-a era eficaz para eliminar os niveis tônicos de $\mathrm{LH}^{6,7}$, reduzindo assim a incidência de más respondedoras ${ }^{8,9}$ entre as mulheres submetidas a tratamento por meio das técnicas de reprodução assistida. Também, desapareceram os ciclos perdidos por ovulação espontânea, pois ocorre uma inibição da função hipofisária responsável pelo pico do $\mathrm{LH}$, fazendo com que a incidência de cancelamento de ciclos para hiperestimulação ovariana controlada (HOC) diminuísse de forma significativa.

$\mathrm{O}$ GnRH é um decapeptídio produzido pelo núcleo arqueado do hipotálamo. Os aminoácidos das posições 6 e 7 são os mais vulneráveis à ação das endopeptidases hipofisárias. Assim, a degradação do GnRH ocorre habitualmente pela quebra da ligação da cadeia entre os aminoácidos 6 e 7 e entre 9 e 10 . Os aminoácidos 2 e 3 (histidina e triptofano) são os responsáveis pela ativação dos receptores hipofisários. Para produzir a liberação máxima de gonadotrofinas é suficiente que o GnRH ocupe apenas $10 \%$ dos seus receptores hipofisários, sendo que cada célula gonadotrófica hipofisária contém aproximadamente 10.000 receptores de GnRH. Depois que as moléculas de GnRH se unem a seus receptores, o complexo GnRH-receptor passa de um a outro sobre a superficie celular. Esta polarização conduz a micro-agregação de complexos-receptor, que amplifica a ação do GnRH sobre a sintese/liberação das gonadotrofinas. Depois da ativação do processo intracelular, o GnRH e os receptores são degradados ou reinseridos na membrana celular ${ }^{10}$.

Os análogos agonistas (GnRH-a) são sintetizados por substituição do aminoácido 6 da cadeia do GnRH por um D-aminoácido e também pelo agrupamento de um radical lamida $\left(-\mathrm{CONH}_{2}\right)$ ao aminoácido 10. No caso específico da goserelina, este GnRH é modificado nos aminoácidos da posição 6 para D.Ser ( $t \mathrm{Bu})$ e 10 para Aza-Gly. Esta substituição provoca uma diminuição na velocidade da sua degradação pelas peptidases hipofisárias, resultando em uma ligação ao receptor mais duradoura e um estímulo mais prolongado para a liberação de LH e de hormônio folículo estimulante (FSH). Devemos lembrar que os agonistas foram desenvolvidos originariamente para serem utilizados no tratamento da anovulação como indutores. Porém, como conseqüência, estes agonistas provocaram efeitos bloqueadores, já que estimulam inicialmente a liberação de LH e FSH, mas sua administração contínua provoca o fenômeno de dessensibilização ou "down-regulation", descrito anteriormente, com a diminuição das gonadotrofinas hipofisárias ${ }^{11,12}$.

O objetivo deste trabalho é estudar os efeitos da goserelina de depósito como agonista do GnRH para a supressão hipofisária, durante a hiperestimulação ovariana controlada (HOC) em fertilização in vitro e transferência de embriões (FIVeTE).

\section{Pacientes e Métodos}

Foram estudados prospectivamente 110 ciclos de FIVeTE, no período compreendido entre junho de 1995 e abril de 1996, envolvendo 101 mulheres regularmente cadastradas no Serviço de Esterilidade Conjugal da Divisão de Reprodução Humana do Centro de Referência da Saúde da Mulher e de Nutrição, Alimentação e Desenvolvimento Infantil (CRSMNADI). A idade das mulheres deste grupo variou de 23 a 42 anos, média de 36,7 anos.

As principais indicações do procedimento quanto a causa da esterilidade estão contidas na Tabela 1.

Tabela 1 - Indicações para a realização da FIV.

\begin{tabular}{lcc}
\hline Indicação & $\mathbf{n}$ & $\%$ \\
\hline Fator tuboperitoneal & 76 & 75,2 \\
Endometriose & 11 & 10,9 \\
Fator ovulatório & 8 & 7,9 \\
Fator masculino & 3 & 3,0 \\
Esca & 3 & 3,0 \\
Total & 101 & 100 \\
\hline
\end{tabular}

A goserelina de depósito (Zoladex ${ }^{\circledR}$ - Zeneca) foi administrada em dose única, para algumas mulheres na fase folicular ( 87 ciclos, $79,1 \%$ ), e para outras, na fase lútea tardia (23 ciclos, 20,9\%); em ambas as situações a administração do medicamento foi precedida por ultra-sonografia endovaginal de controle. Após 15 a 20 dias realizouse nova ultra-sonografia endovaginal para iniciar a HOC, tendo como critérios de inclusão endométrio com padrão linear e ausência de cistos ovarianos com diâmetro maior que $30 \mathrm{~mm}$. Na presença de cistos maiores, fazia-se a aspiração com agulha própria, sob visão ultra-sonográfica, após o que se iniciava a HOC. 
O protocolo de HOC utilizado compunha-se de administração IM de 300 UI de hMG (Pergonal® - Serono) diariamente. O primeiro controle ultrasonográfico era realizado no $5^{\circ}$ dia de indução e após, a intervalos variáveis, conforme a necessidade de cada caso, até que se observassem pelo menos dois folículos com diâmetro maior ou igual a $18 \mathrm{~mm}$. Neste momento era programado o horário da administração de 10.000 UI de hCG (Profasi ${ }^{\circledR}$ - Serono), sendo que 35 horas após esta injeção realizava-se a aspiração folicular.

A aspiração folicular era realizada em centro cirúrgico, respeitando-se todos os preceitos quanto à anti-sepsia necessária para tal procedimento. Utilizamos um aparelho Aloka $500 \AA \mathrm{com}$ transdutor endovaginal de $3.5 \mathrm{MHz}$, revestido por condom estéril e, sobre este, uma guia de agulha específica para punção.

Os oócitos recuperados foram inseminados, em média, duas horas após a aspiração folicular e 20 horas depois realizava-se a verificação da fertilização: fertilizados viáveis (2 pronúcleos), fertilizados inviáveis (poliplóides, degenerados, etc.) ou não-fertilizados (ausência de pronúcleos). Após 72 horas da aspiração folicular se transferiam os embriões clivados para o interior da cavidade uterina. Utilizamos para a transferência embrionária o catéter de Frydman (CCD ${ }^{\circledR}$ - França).

A suplementação hormonal da fase lútea era realizada a partir da transferência dos embriões, de duas maneiras distintas: para as pacientes com até 10 oócitos aspirados, utilizamos 2.000 UI de hCG por via IM a cada 3 dias, e para as pacientes com mais de 10 oócitos aspirados e/ou obesas e/ou com menos de 30 anos e/ou hirsutas, administrávamos $50 \mathrm{mg}$ de progesterona em óleo (Progesterone ${ }^{\circledR}$ Eli Lilly) diariamente ou comprimidos de progesterona (Utrogestan ${ }^{\circledR}$ - Bensis Iscovesco), 600 $\mathrm{mg} /$ dia sendo administrados 1 comprimido via oral a cada 8 horas ou, por via vaginal, 2 comprimidos à noite e um comprimido pela manhã.

O controle após a transferência de embriões fazia-se aproximadamente após 15 dias, com a dosagem sangüinea quantitativa do beta hCG. Uma vez que o teste resultasse positivo, realizava-se um controle ultra-sonográfico a cada 15 dias. A suplementação da fase lútea, nestes casos, se estendia até a $12^{a}$ semana de gestação. Após este período, encaminhávamos a paciente para um serviço de acompanhamento pré-natal.

\section{Resultados}

Foram estudados 110 ciclos de FIVeTE em 101 pacientes, sendo que 28 destes ciclos $(25,5 \%)$ foram cancelados pelas seguintes causas: falha de bloqueio - 2 casos (7,1\%); má resposta ao estímulo ovariano - 25 casos (89,3\%); hipertensão arterial grave - 1 caso $(3,6 \%)$.

Foi necessário executar aspiração de cistos ovarianos antes do início da HOC em 7 casos, sendo que em todos estes casos a goserelina de depósito foi administrada na fase folicular $(6,4 \%$ do total de ciclos; $30,4 \%$ dos casos de administração em fase folicular).

Dos 82 ciclos que terminaram em aspiração folicular em situação adequada, foram recuperados 683 oócitos, de 0 a 33 oócitos por aspiração, resultando em média 7,8 oócitos/paciente. Dos 683 oócitos inseminados, 456 foram fertilizados, observando-se uma taxa de fertilização de $66,8 \%$, portanto. Houve clivagem em 406 oócitos fertilizados $(89,0 \%)$ e 50 oócitos não clivaram $(11,0 \%)$. Foram transferidos de 1 a 5 embriões/paciente, sendo que a média foi de 3,3 embriões por paciente.

Em 12 ciclos não houve transferência $(14,6 \%)$, sendo que das 70 transferências realizadas $(85,4 \%)$, foram obtidas 16 gestações clínicas (taxa de gravidez: $22,85 \%)$.

O resumo dos resultados obtidos encontramse na Tabela 2.

Tabela 2 - Resumo dos principais índices observados

\begin{tabular}{lc}
\hline & $\%$ \\
\hline Cancelamentos & 25,5 \\
Fertilização & 66,8 \\
Clivagem & 89,0 \\
Transferência & 85,4 \\
Gravidez & 22,8 \\
\hline
\end{tabular}

\section{Discussão}

A literatura mostra uma taxa variável de ciclos cancelados no uso de GnRH-a. Ferrier et al. ${ }^{10}$ referem taxa de cancelamento de $23,7 \%$, Barri et al. ${ }^{11} 31,57 \%$ com uso de análogo de administração nasal e $13,6 \%$ quando administrado por via subcutânea. Não temos dúvidas de que uma das causas desta taxa de $25,5 \%$ é fato de lidarmos algumas vezes com pacientes com idade avançada, levando portanto a um alto índice de cancelamentos por má resposta ao estímulo ovariano.

Chamou-nos a atenção o aparecimento de 7 casos de pacientes com cisto ovariano prévio à HOC, justamente as pacientes que receberam o análogo na fase proliferativa do ciclo. Sampaio et al. ${ }^{12}$, comparando o protocolo curto (fase folicular) com o protocolo longo (fase lútea), observaram maior freqüência de cistos quando da utilização do protocolo curto (23\%), sendo que no protocolo longo o aparecimento de cistos ocorreu em 10\% 
dos casos. Também neste trabalho, demonstrouse não haver influência destes sobre os resultados da FIV, o que também foi demonstrado por outros autores ${ }^{13,14,15}$.

Oyesanya et al. ${ }^{16}$ relatam em seu trabalho a preferência pela administração do análogo de depósito na fase folicular, pois não haveria o risco de uma administração inadvertida durante uma gestação ainda desconhecida.

Quanto à taxa de oócitos recuperados por paciente, o valor de 7,8 está em concordância com outros trabalhos, como o de Lockwood et al. ${ }^{17}$ que comparando vários análogos com protocolos diferentes de FIV, encontraram valores de 6,4 a 7,3 de oócitos/paciente. Tanto a taxa de fertilização quanto a taxa de clivagem não diferem das encontradas na literatura ${ }^{16,17,18}$.

Em estudo prévio, Matta et al. ${ }^{19}$ mostraram que o efeito bloqueador de uma única dose de goserelina de depósito dura em torno de 5 semanas. Portanto, muitas vezes este efeito se estende à fase lútea precoce, fazendo com que seja imperioso o suporte hormonal após a transferência embrionária.

A taxa de gravidez encontrada por nós, de $22,85 \%$ (16 gestações), tem um valor compativel com outros trabalhos. Tapanainen et al. ${ }^{20}$, em trabalho comparando o uso de goserelina de depósito e buserelina, obtiveram no primeiro grupo uma taxa de gravidez de $22,4 \%$, ao passo que no segundo grupo houve uma taxa de 24,0\%, concluindo que a goserelina de depósito, administrada por via subcutânea, oferece uma alternativa muito útil para o processo de supressão hipofisária em FIVeTE.

Outros autores, como Ruiz Velasco et al. ${ }^{21}$, também descrevem bons resultados usando esquema semelhante (goserelina de depósito +hMG +hCG) em 18 casos, dos quais 3 não responderam aos medicamentos para o estímulo ovariano e dos 15 casos restantes, sendo 8 tranferências intrafalopianas de gameta (GIFT), 4 FIVeTE e 2 inseminações intra-uterinas (IIU), resultaram 8 gestações $(57,1 \%)$ na primeira tentativa de tratamento.

Concluímos que a utilização da goserelina de depósito em ciclos de HOC para FIVeTE mostrase promissora, uma vez que não compromete os resultados obtidos, com indice de sucesso semelhante ao observado na literatura, enquanto permite a redução do número de aplicações de drogas envolvidas na HOC, fato de extrema importância num serviço público como o CRSMNADI.

\section{SUMMARY}

Purpose: to verify the effects of the goserelin depot as GnRH agonist for hypophysis suppression, during the controlled ovarian hyperstimulation ( $\mathrm{COH}$ ) for in vitro fertilization and intrauterine embryo transfer (IVF \& ET).

Method: this is a prospective study of 110 cycles of 101 women. Goserelin depot was administered subcutaneously as a single dose; for some women (87 cycles) it was administered in the first phase of the menstrual cycle, and in 23 cycles it was administered in the luteal phase. The administration of menopausal gonadotropins was daily, until the identification of at least two follicles with a diameter equal to or larger than $18 \mathrm{~mm}$; at this time the chorionic gonadotropin was administered and the follicular aspiration was programmed. Results: the women's age average was 36.7 years (between 23 to 42 years). The main indications for IVF \& ET were: tubo-peritoneal factor (75.2\%), endometriosis (10.9\%), ovulatory factor (7.9\%), male factor (3.0\%) and unexplained infertility (3.0\%). Of the total of the cycles, $28(25.5 \%)$ cycles were cancealed. In 7 cycles (8.04\%), for which goserelin depot was administered in the first phase of the menstrual cycle, it was necessary to perform the ovarian cysts aspiration before beginning the ovulation induction. On the average, 3.3 embryos were transferred for each patient (1-5 embryos per woman). Of the total of 70 embryos transferred, 16 clinical pregnancies resulted (pregnancy rate: $22.85 \%$ ).

Conclusion: the goserelin depot administration is a useful alternative for pituitary suppression for IVF \& ET, since its results are similar to those observed in the literature, and the patient does not need to come every day to receive medication, a fact of extreme importance in a public service.

KEY WORDS: Ovulation. Ovulation Induction. Gonadotropins. Fertilization in vitro. Embryo Transfer.

\section{Referências}

1. Rabin D, McNeil L W. Pituitary and gonadal desensitization after continuous luteinizing hormone-releasing hormone infusion in normal females. J Clin Endocrinol Metab 1980; 51:873-6.

2. Howles C M, Macnamee MC, Edwards RG, Goswamy $\mathrm{R}$, Steptoe PC. Effect of high tonic levels of luteinizing hormone on outcome of in-vitro fertilization. Lancet 1986; 2:521-2.

3. Van Uem JF, García JE, Liu HC, Rosenwaks Z. Clinical aspects with regard to the occurrence of an endogenous LH surge in gonadotropin induced normal menstrual cycles. J In Vitro Fertil Embryo Transfer 1986; 3:345-9.

4. Fleming R, Coutts JRT. Induction of multiple follicular growth in normally menstruating women with endogenous gonadotropin suppression. Fertil Steril 1986; 45:226-30. 
5. Pellicer A, Lightman A, Diamond MP, Russell JB, De Cherney AH. Outcome of in vitro fertilization in women with low response to ovarian stimulation. Fertil Steril 1987; 47:812-5.

6. Porter RN, Smith W, Craft IL. Induction of ovulation for in vitro fertilization using buserelin and gonadotrophins. Lancet 1984; 2:1284-5.

7. Macnamee M, Howles CM, Edwards RG. Pregnacies after IVF when high tonic LH is reduced by longterm treatment with GnRH agonists. Hum Reprod 1987; 2:569-71.

8. Neveu S, Hedon B, Bringer J, Chinchole JM, Arnal J, Humeau C, et al. Ovarian stimulation by a combination of gonadotropin releasing hormone agonist and gonadotropins for in vitro fertilization. Fertil Steril 1987; 47:639-43.

9. Smitz J, Devroey P, Braeckmans P, Camus M, Khan I, Staessen C, et al. Management of failed cycles in an IVF/GIFT programme with the combination of a GnRH analogue and HMG. Hum Reprod 1987; 2:309-14.

10. Ferrier A, Rasweiler JJ, Bedford JM, Prey K, Berkeley AS. Evaluation of leuprolide acetate and gonadotropins versus clomiphene citrate and gonadotropins for in vitro fertilization or gamete intrafallopian transfer. Fertil Steril 1990; 54:90-5.

11. Barri PN, Martínez F, Coroleu B, Veiga A, Calderón G: Experiencia clinica de la utilización de agonistas del Gn-RH en un programa de fertilizacion in vitro (FIV). Drugs Today 1988; 24:51-60.

12. Sampaio M, Serra V, Miró F, Calatayud C, Castellví RM, Pellicer A. Development of ovarian cysts during gonadotrophin-releasing hormone agonists (GnRHa) administration. Hum Reprod 1991; 6:194-7.

13. Ron-El R, Herman A, Golan A, Raziel A, Soffer Y, Caspi E. Follicle cyst formation following longacting gonadotropin-releasing hormone analog administration. Fertil Steril 1989; 52:1063-6.

14. Fedberg D, Ashkenazi J, Dicker D, Yeshaya A,
Goldman GA, Goldman JÁ. Ovarian cyst formation: a complication of gonadotropinreleasing agonist therapy. Fertil Steril 1989; $51: 42$.

15. Herman A, Ron-El R, Golan A, Nahum H, Soffer Y, Caspi E. Follicle cysts after menstrual versus midluteal administration of gonadotropinreleasing hormone analog in in vitro fetilization. Fertil Steril 1990; 53:854-8.

16. Oyesanya OA, Teo SK, Quah E, Abdurazak N, Lee FY, Cheng WC. Pituitary down-regulation prior to in-vitro fertilization and embryo transfer: a comparison between a single dose of Zoladex depot and multiple daily doses of Suprefact. Hum Reprod 1995; 10:1042-4.

17. Lockwood GM, Pinkerton SM, Barlow DH. A prospective randomized single-blind comparative trial of nafarelin acetate withe buserelin in longprotocol ganadotropin-releasing hormone analogue controlled in-vitro fertilization cycles. Hum Reprod 1995; 10:293-8.

18. Testart J, Forman R, Belaisch-Allart J, Volante M, Hazout A, Strubb N, et al. Embryo quality and uterine receptivity in in-vitro fertilization cycles with or without agonists of gonadotropin-releasing hormone. Hum Reprod 1989; 4:198-201.

19. Matta WH, Shaw RW, Burford GD. Endocrinologie and clinical evaluation following a single administration of a gonadotropin releasing hormone agonist (Zoladex) in a depot formulation to premenopausal women. Fertil Steril 1988; 49:163-5.

20. Tapanainen J, Hovatta O, Juntunen K, Martikainen H, Ratsula K, Tulppala M, et al. Subcutaneous goserelin versus intranasal buserelin for pituitary down-regulation in patients undergoin IVF: a randomized comparative study. Hum Reprod $1993 ; 8: 2052-5$

21. Ruiz Velasco V, Allende S. Goserelin followed by assisted reproduction: results in infertile women with endometriosis. Int $\mathrm{J}$ Fertil Womens Med 1998; 43:18-23 